\title{
Multimode metal-lined capillaries for Raman collection and sensing
}

\author{
Michael P. Buric,,$^{1,2}$ Kevin P. Chen, ${ }^{1,2}$ Joel Falk, ${ }^{1,2, *}$ and Steven D. Woodruff ${ }^{1}$ \\ ${ }^{1}$ National Energy Technology Laboratory, 3610 Collins Ferry Rd., Morgantown, West Virginia, 26507, USA \\ ${ }^{2}$ Department of Electrical and Computer Engineering, University of Pittsburgh, 348 Benedum Hall, Pittsburgh, \\ Pennsylvania 15261, USA \\ *Corresponding author: falk@engr.pitt.edu
}

Received July 19, 2010; revised September 24, 2010; accepted October 12, 2010; posted October 18, 2010 (Doc. ID 131862); published November 11, 2010

Reflective metal-lined capillary waveguides are useful for laser-power delivery or for collecting scattered light in sensing applications. We theoretically examine the multimode propagation of polarized light in largediameter, metallized, capillary waveguides using a new perturbation technique valid for all waveguide modes. This modeling permits prediction of the collection efficiency of Raman or fluorescent light produced in the waveguide at all angles. These theoretical results are supported by measuring the intensity and angular distribution of collected scattered gas-Raman Stokes power. (C) 2010 Optical Society of America

OCIS codes: $300.6450,230.7370,280.4788$.

\section{INTRODUCTION}

Large-core-diameter waveguides are important in a number of applications including delivering high-power laser light to a target and collecting scattered light for sensing [1-8]. For visible-wavelength power-delivery applications, solid-core dielectric waveguides (such as silica multimode fibers) are often appropriate. However, in the mid-ir where glass-fiber cores are generally not transparent, hollow core waveguides are necessary. It is often advantageous to employ metal-lined or metal/dieletric-lined waveguides to boost hollow-core guide-wall reflectivity and resulting waveguide transmission. Previous studies have shown that these waveguides, used for sensing applications, offer improved light-collection abilities versus traditional spatially unconfined light-collection geometries [9-12].

Currently, metal-lined, hollow-core, waveguides (or metal-lined guides with an inner dielectric lining) that exhibit low losses at optical wavelengths are commercially available and being used as both a power delivery and a light-collection apparatus [9,10,13]. Hollow-core, dielectric clad waveguides (without a metal lining) are also of limited use for these applications. Because dielectric claddings generally have refractive indices greater than unity, high reflectivity (and high waveguide transmission) occurs only for the very few modes in the metal-free guide that propagate near grazing incidence to the guide walls $[11,14]$.

Recently, we detailed the possibility of using metal- or metal/dielectric-lined waveguides as efficient Raman sensing cells for gasses [9]. Therein, analyte gases are introduced into the capillary waveguide along with a visible or near-ir wavelength pump beam. The metal-lined capillary is used to propagate both the pump beam and the emitted Stokes radiation, so that Stokes radiation can be transferred to the end of the guide where it is collected and analyzed. Measurement of the Stokes wavelength and its intensity permits determination of the composition and density of the gaseous analyte flowing inside the capillary. This technique has been shown to be more effective than unconfined Raman collection [15], but the exact theoretical magnitude of collection improvement has been unknown because of the complicated multimode characteristics of metal-lined capillaries. In order to detail precisely the Raman optical power that can be collected using a metal-lined capillary waveguide, we must first know the loss coefficient of each mode that can propagate in the guide. Then, we can infer a total collectible power for a given detected solid angle and waveguide length.

In this paper we calculate the modes that can propagate in such a waveguide. This work builds upon theory developed in the 1940s-1960s [16,17] and extends it to predict the loss of high-order modes propagating at large angles that are useful for collection of Raman or fluorescent scattered light. Our extensions to this earlier work incorporate a computer-based calculation to allow the accurate determination of the loss of each of hundreds of propagating modes. This complexity is necessary for finding the power collected in any scattering process, where higher-order modes can contribute significantly to the total collected power. Previously, simplifications have allowed closed-form, approximate solutions to be found for low-order modes in waveguides of this type $[17,18]$. Here, we find more accurate solutions for all possible modes that can propagate in the waveguide. A typical scattering process contributes equal power to each mode, and this calculation allows an accurate estimation of scattered light propagating inside a hollow waveguide. We use this theory to calculate the total spontaneous Raman power that can be collected using a metal-lined guide filled with a Raman-active gas (with a low depolarization ratio) and compare these results to measurements of collected Raman power. We show that the introduction of the metal coating greatly reduces waveguide losses and greatly im- 
proves Raman collection efficiency over that available from a hollow-core, purely dielectric capillary.

A central result of our work is that higher-order propagating modes of the Raman scattered light make significant contributions to the total Raman power collected. This occurs in spite of the large absorption coefficients associated with these modes. In essence, because Raman scattered light produces a source that is distributed through the guide, all light generated near the fiber's output is not strongly attenuated and contributes to the Raman output and to the large angular distribution of the Raman signal.

\section{THEORY}

Figure 1 is a schematic diagram of a metal-lined waveguide. The waveguide is constructed from a bare-silicaglass capillary tube that has been internally coated with a reflective metal lining. Previous researchers have shown that the internal deposition of silver, aluminum, or gold can be accomplished using various processes. Highquality internal coatings have been produced using metal oxide chemical vapor deposition or liquid reaction deposition $[6,19]$. Low-loss waveguides of these types with internal diameters ranging from about $250 \mu \mathrm{m}$ to over $1 \mathrm{~mm}$ have been reported. In some cases, an additional internal, transparent, dielectric coating is provided to protect the metal coating from oxidation or wear and to increase transmission. Herein we will not attempt to account for additional internal coatings or multi-layer dielectrics, although they have been studied in detail previously $[1,2,7]$. The use of a dielectric lining can lead to a significant reduction in losses for all modes, although some fabrication difficulties have been noted in the production of internal dielectric-over-metal linings designed for visible transmission [19-24]. Furthermore, these dielectric linings have been shown to significantly reduce bending losses. In our Raman sensor application, it is easy to support the waveguide to avoid such bending losses, making additional internal-dielectric linings somewhat less important.

To determine the propagating waveguide modes, we write general expressions for the fields in the core and in the metal cladding and equate the tangential components of electric and magnetic fields at each side of the boundary at $r=a$. This leads to a transcendental characteristic equation that implicitly describes all modes that can propagate in the guiding structure. All fields in the core as well as in the cladding can be written in terms of the longitudinal field components $\mathrm{E}_{\mathrm{z}}$ and $\mathrm{H}_{\mathrm{z}}$. Textbook studies of circularly symmetric structures [16] have shown that field components in the air core can be described in

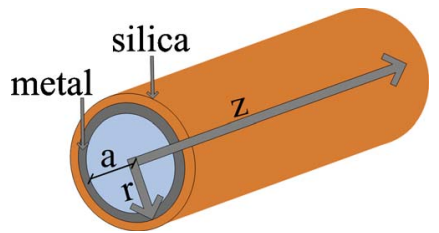

Fig. 1. (Color online) Cylindrical, metal-lined waveguide. The radius of the waveguide is $a$; the distance from the center of the guide is denoted by $r$, and the direction of propagation is $z$. terms of $J_{n}$, Bessel functions of the first kind. The field components can be described in cylindrical coordinates $(r, \theta, z)$ as

$$
E_{z}^{i}=\sum_{n} a_{n}^{i} J_{n}\left(k_{i} r\right) e^{i(\gamma z+n \theta)} \quad \text { and } \quad H_{z}^{i}=\sum_{n} b_{n}^{i} J_{n}\left(k_{i} r\right) e^{i(\gamma z+n \theta)},
$$

where $k_{i}$ is the (complex) transverse eigenvalue in the core, $\gamma$ is the (complex) longitudinal eigenvalue, and $a_{n}^{i}$ and $b_{n}^{i}$ are expansion coefficients. Inside the metal, the $E_{Z}$ and $H_{Z}$ fields can be written in terms of $H_{n}^{(1)}$, Hankel functions of the first kind:

$E_{z}^{e}=\sum_{n} a_{n}^{e} H_{n}^{(1)}\left(k_{e} r\right) e^{i(\gamma z+n \theta)}$ and $H_{z}^{e}=\sum_{n} b_{n}^{e} H_{n}^{(1)}\left(k_{e} r\right) e^{i(\gamma z+n \theta)}$,

where $k_{e}$ is the (complex) transverse eigenvalue inside the metal lining, and $a_{n}^{e}$ and $b_{n}^{e}$ are expansion coefficients. The subscripts $i$ and $e$ denote fields inside the air-core and fields external to the core-i.e., in the metal. Figure 2 shows the relationships between the mode-constants. The magnitude of the wave-vector is: $k=2 \pi / \lambda$ where $\lambda$ is the free-space wavelength of the propagating modes. The dielectric constant in the core is 1.0 and the complex dielectric constant in the metal is given by $\nu$. The vector diagram allows us to infer relationships between the wave vectors in the core and in the metal. In particular, we can write

$$
\begin{aligned}
& k_{e}=\sqrt{\nu^{2} k^{2}-\gamma^{2}}, \\
& k_{i}=\sqrt{k^{2}-\gamma^{2}} .
\end{aligned}
$$

Equating the tangential components of the electric and magnetic fields at the air-metal boundary produces a set of four continuity equations. For each integer $n$, a solution to these equations requires

$$
\begin{gathered}
{\left[\frac{1}{k_{i m} a} \cdot \frac{J_{n}{ }^{\prime}\left(k_{i m} a\right)}{J_{n}\left(k_{i m} a\right)}-\frac{1}{k_{e m} a} \cdot \frac{H_{n}{ }^{(1) \prime}\left(k_{e m} a\right)}{H_{n}{ }^{(1)}\left(k_{e m} a\right)}\right] \cdot\left[\frac{k^{2}}{k_{i m} a} \frac{J_{n}{ }^{\prime}\left(k_{i m} a\right)}{J_{n}\left(k_{i m} a\right)}\right.} \\
\left.-\frac{k^{2} \nu^{2}}{k_{e m} a} \cdot \frac{H_{n}^{(1) \prime}\left(k_{e m} a\right)}{H_{n}{ }^{(1)}\left(k_{e m} a\right)}\right]=n^{2} \gamma_{m}{ }^{2}\left[\frac{1}{\left(k_{e m} a\right)^{2}}-\frac{1}{\left(k_{i m} a\right)^{2}}\right]^{2} .
\end{gathered}
$$

Thus each integer $n$ produces $m$ mode-solutions. The prime symbol is used to indicate the derivatives of functions. Equation (5) is termed the characteristic equation

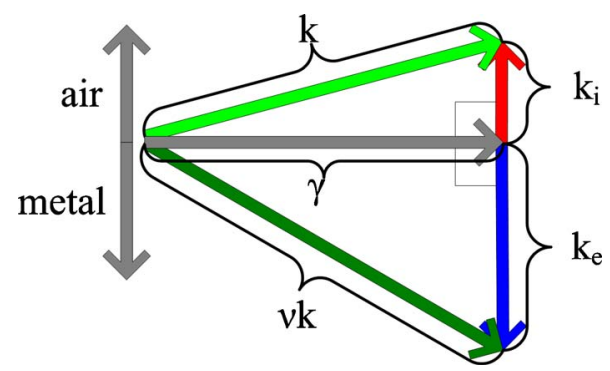

Fig. 2. (Color online) Mode vector diagram at air/metal interface. 
for the $m$ th mode. The solutions of this equation can be used to describe any propagating mode in any large cylindrical guide [16-18,25].

Equation (5) is greatly simplified by noting several physical constraints. First, in this work we are interested only in polarized propagating modes. Our experimental objective is to use the capillary to collect Stokes Raman radiation produced by a linearly polarized laser beam interacting with gases with low depolarization ratios. Thus the Stokes Raman light is then also highly polarized, and very little optical power couples into un-polarized modes. The only set of cylindrical modes that exhibits linear polarization is the hybrid $E H_{n m}$ mode set with $n=1$ [26]. Considering all of the modes in the $E H_{1 m}$ set will allow us to characterize losses in modes propagating at higher angles of incidence, i.e., filling more of the waveguide's angular aperture. Although the $E H_{10}$ mode has been widely studied because it provides the lowest possible transmission loss using a metal/dielectric-lined capillary, the $T E$ modes have lower losses in purely metal-lined capillaries [17]. However, these modes are not linearly polarized and are of little interest for the present work.

Second, we can assume that the capillary's diameter is large in comparison to the wavelength of the propagating light. This implies that $\left|k_{e m} a\right| \approx 2 \pi a / \lambda|\nu| \gg 1$ and therefore the Hankel functions in Eq. (5) can be approximated by their asymptotic values:

$$
\frac{H_{1}^{(1) \prime}\left(k_{e m} a\right)}{H_{1}^{(1)}\left(k_{e m} a\right)} \approx i .
$$

All values of $k_{e m} a$ evaluated in this paper satisfy this approximation.

Finally, we observe that for all propagating modes in silver- or aluminum-lined capillaries

$$
\left|\frac{k_{i m}}{k_{e m}}\right| \ll 1,
$$

and consequently we can ignore all terms containing powers of $k_{i m} / k_{e m}$ that are greater than 1.

Setting $n=1$ in Eq. (5) and using the approximations enumerated in Eqs. (6) and (7) produces a significantly simplified characteristic equation:

$$
J_{0}\left(k_{i m} a\right)=\left[\frac{1 / 2\left(\nu^{2}+1\right)}{\sqrt{\nu^{2}-\frac{\gamma_{m}^{2}}{k^{2}}}} \cdot \frac{i k_{i m}}{k}+\frac{1}{k_{i m} a}\left[1-\frac{\gamma_{m}}{k}\right]\right] J_{1}\left(k_{i m} a\right)
$$

Equation (8) can be solved using a new perturbation technique that accurately determines losses in higher-order modes. Previous authors' simplified solutions of Eq. (8) have assumed that $\gamma_{m} \approx k$, and involve only a single-step perturbation, which is adequate only for low-order modes $[17,18]$. Our solution will conversely also produce accurate solutions for higher-order mode losses. Therein, we note that the bracketed term on the right-hand-side of Eq. (8) is generally small and $0<\left|J_{1}\left(k_{i m} a\right)\right|<1$. Thus a firstorder solution to Eq. (8) is $k_{i m} a \approx u_{i m}$, where $u_{i m}$ is the real-valued $m$ th zero of the $J_{0}$ Bessel function [27]. The zeros of the zeroth -order Bessel function are well known and can be calculated with standard numerical packages or can be accurately estimated using

$$
u_{1 m} \approx(m-1 / 4) \pi
$$

We note that if $k_{i m} a \equiv u_{i m}$, then Eq. (4) implies that $\gamma_{m}$ is real and the mode has no loss. (If a solution for $k_{i m}$ is found, $\gamma_{\mathrm{m}}$ and $k_{e m}$ are derived from Eqs. (4) and (3), respectively.)

To find a perturbative correction to a solution of Eq. (8), we make an initial guess $k_{i m} a \approx u_{i m}$ and use that value to evaluate the (small) right-hand side of Eq. (8). After determining the right-hand side of Eq.(8), a better solution for $k_{i m} a$ can be found numerically. The perturbation technique assumes that each exact solution for $k_{i m} a$ will be relatively close to $u_{1 m}$ (the lossless zeroth-order solution) and will be given by

$$
k_{i m} a=u_{1 m}+\Delta
$$

where $\Delta$ is the complex deviation from the Bessel function zero. We solve for $\Delta$ by performing a Taylor-series expansion for $J_{0}$ and $J_{1}$ in Eq. (8). The resulting expression for $\Delta$ is

$$
\Delta=\frac{J_{1}\left(k_{i m} a\right)\left[\frac{\left(\nu^{2}+1\right) \cdot i}{2 \sqrt{\nu^{2}-1+\left(\frac{k_{i m}}{k}\right)^{2}} \cdot \frac{k_{i m}}{k}+\frac{1}{k_{i m} a}\left[1-\sqrt{1-\left(\frac{k_{i m}}{k}\right)^{2}}\right]-J_{0}\left(k_{i m} a\right)}\right.}{-J_{1}\left(k_{i m} a\right)-\left(J_{0}\left(k_{i m} a\right)-\frac{J_{1}\left(k_{i m} a\right)}{k_{i m} a}\right)\left[\frac{\left(\nu^{2}+1\right) \cdot i}{2 \sqrt{\nu^{2}-1+\left(\frac{k_{i m}}{k}\right)^{2}} \cdot \frac{k_{i m}}{k}+\frac{1}{k_{i m} a}\left[1-\sqrt{1-\left(\frac{k_{i m}}{k}\right)^{2}}\right]}\right.} .
$$

Figure 3 is a flow-chart illustrating how our perturbative technique is used iteratively to find precise solutions to the characteristic equation. First, an initial guess for $k_{i m} a$ consists of the exact zero of the zeroth-order Bessel function. Then, the initial value of $\Delta$ is determined from Eq. (11). The initial value of $\Delta$ is added to the initial value of 


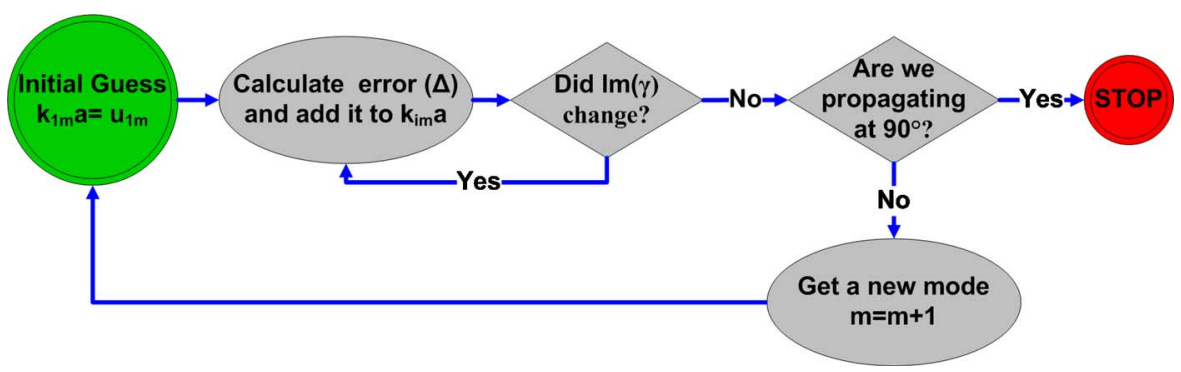

Fig. 3. (Color online) Iterative algorithm for finding propagating mode losses.

$k_{i m} a$ to find a solution closer to the exact value than the initial guess, and the process is repeated with a new value of $\left(k_{\text {im }} a\right)_{\text {new }}=\left(k_{\text {im }} a\right)_{\text {old }}+\Delta$. For each iteration, the value of $k_{i m}$ is used to find $\gamma_{m}$. The imaginary part of $\gamma_{m}$ determines the loss coefficient. When the change in the $\operatorname{Im}\left(\gamma_{m}\right)$ is less than $10^{-5} \mathrm{~m}^{-1}$ from the previous iteration, the process is stopped, and the values of $k_{i m}$ and $\gamma_{m}$ for the $m$ th mode are calculated.

After $k_{i m}$ has been found for a particular mode, the next higher-order mode is sought by re-initializing the value of $k_{i(m+1)} a$ to that of the next Bessel function zero, or $u_{1, m+1}$. As each new mode is calculated, the angle of propagation of that mode is determined. When the propagation angle is close to $90^{\circ}$, we have found all of the modes that can propagate in a particular guide, and the algorithm is complete.

The longitudinal propagation constant $\left(\gamma_{m}\right)$ is determined from Eq. (4) and can be used to find the propagation angle $\left(\theta_{m}\right)$ and power-loss coefficient $\left(\alpha_{m}\right)$ for each mode:

$$
\begin{gathered}
\theta_{m}=\cos ^{-1}\left(\operatorname{Re}\left(\frac{\gamma_{m}}{k}\right)\right), \\
\alpha_{m}=2 \operatorname{Im}\left(\gamma_{m}\right) .
\end{gathered}
$$

Figure 4 shows the typical variation of the loss coefficient with propagation angle-i.e., numerical solutions of Eqs. (8), (12), and (13). At any angle, the smaller loss coefficient predicted for the large bore size capillary can be

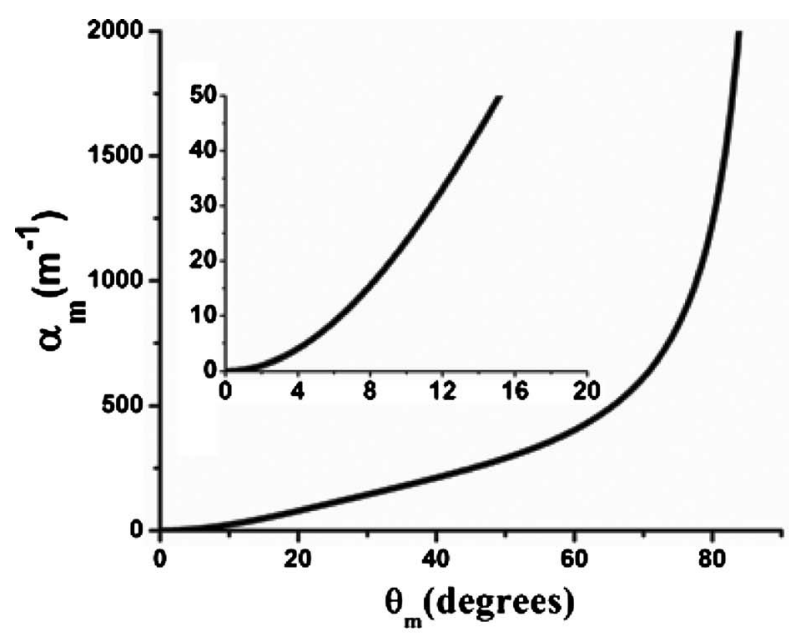

(a) qualitatively understood as a ray making fewer lossy bounces in the large bore size guide as it propagates down the capillary. We note that internal dielectric linings have been shown to significantly reduce losses for each mode in a metal waveguide by the same factor [6,24]. If an appropriate inner-dielectric lining were applied, the curves in the figure would decrease by some constant factor. In practice, most commercially available metal waveguides (including the ones described herein) include an inner dielectric lining. In practice, these dielectric linings, which must be much less than one wavelength thick, are difficult to produce for use in the visible/near-ir spectrum $[1,4,6,28]$.

To verify these loss simulations, a He-Ne laser $(\lambda$ $=632.8 \mathrm{~nm})$ with a $\sim 2 \mathrm{~mm}$ diameter $\left(1 / \mathrm{e}^{2}\right)$ beam was launched into a $530 \mu \mathrm{m}$ I.D. silver-lined $2 \mathrm{~m}$ long capillary using a $3.1 \mathrm{~mm}$ effective focal length (efl) aspheric singlet. This focusing arrangement provides approximately equal power into all modes with angles of propagation up to about $18^{\circ}$ (half-angle). At the other end of the waveguide, a $25 \mathrm{~mm}$ singlet was used to collimate the output. A graduated aperture placed behind the collimating lens and a power meter positioned behind the aperture allowed measurement of output power versus integrated angle. The waveguide was supported and held straight to avoid the introduction of large bending losses common to hollow metalized waveguides. Figure 5 shows the result of this simple experiment. Also shown is a simulation of integrated forward-propagating power that has been scaled to account for detector efficiency and in-

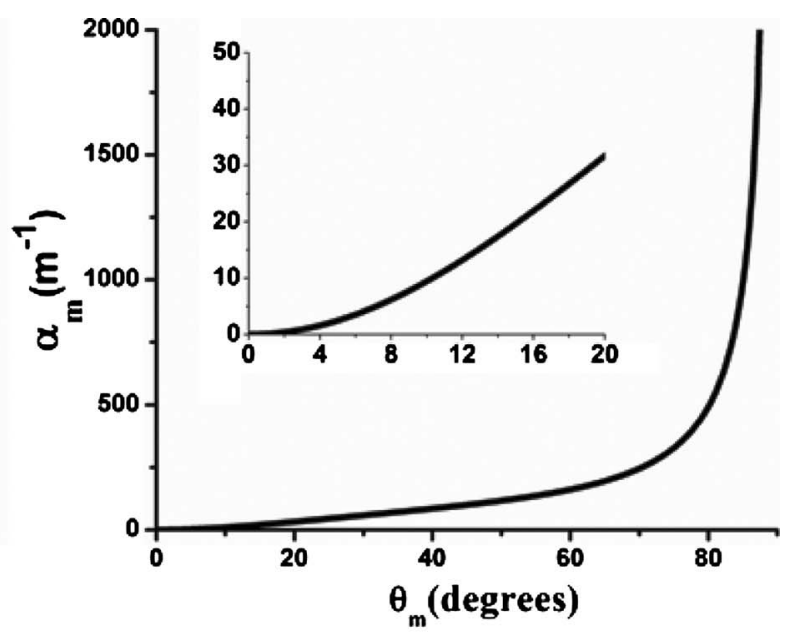

(b)

Fig. 4. Loss coefficient $\alpha_{m}$ as a function of propagation angle $\theta_{m}$; silver-coated capillary $\lambda=584.5 \mathrm{~nm}$; (a) $2 a=320 \mu \mathrm{m}$. (b) $2 a=800 \mu \mathrm{m}$. 


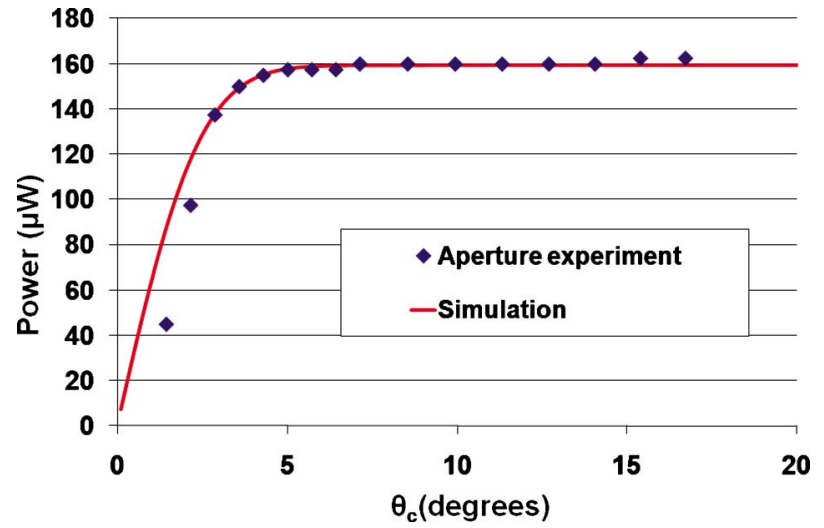

Fig. 5. (Color online) Waveguide power output as a function of collection angle $\left(\theta_{c}\right)$.

put power. The simulations assume that all modes are excited equally and that the loss coefficient for each mode is calculated as described previously-i.e., $\alpha_{m}(\theta)$ is found from Eqs. (8), (12), and (13). (The loss coefficient is written as $\alpha_{m}(\theta)$ to highlight its variation with angle.) The values of $\alpha_{m}(\theta)$ were used to predict the variation of power output as the detected acceptance angle was increased. Measurements were taken up to about $20^{\circ}$ (half-angle) where under-filling of modes at the input to the waveguide was expected to occur. The measured output power correlates well with the theory.

Figure 5 shows the effect of forward wave propagation through a full $2 \mathrm{~m}$ length of the waveguide, where each mode experiences a transmission given by $\mathrm{e}^{(-\alpha \mathrm{l})}$, where $\alpha$ is a function of propagation angle (Fig. 4). Figure 5 implies that modes that propagate through the entire guide with $\theta_{c} \geq 5^{\circ}$ are lost. The total power loss coefficient for propagation at $5^{\circ}$, calculated via the algorithm outlined in Fig. 3, is $4.2 \mathrm{~m}^{-1}$, and thus rays propagating at $5^{\circ}$ to the waveguide's axis have a transmission coefficient of approximately $2.25 \times 10^{-4}$. Later, we will show that despite these large losses, higher-order modes can make significant contributions to total collectible Raman power.

\section{ENHANCED RAMAN SCATTERING COLLECTION USING METAL WAVEGUIDES}

The primary motivation for the work reported in this paper was to understand how hollow-core, metal-lined waveguides could be used as optical cells that both contain Raman-active gases or liquids and enhance the collection of spontaneous Stokes signals from those analytes. We have previously shown that this configuration can be used to collect significantly more Raman light than a diffraction-limited, unconfined configuration. Figure 6 is a diagram of the Raman light collection system described previously $[9,15]$.

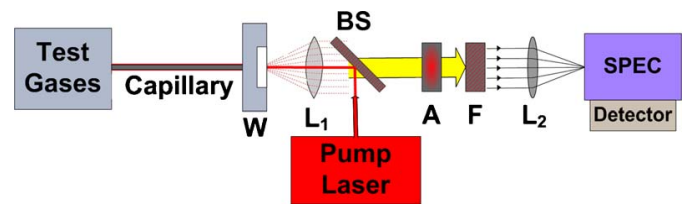

Fig. 6. (Color online) Gas-Raman enhanced collection system.
The optical components therein are arranged similarly to those of a collinear Raman microscope, with the waveguide positioned at the usual sample position [29]. The pump-laser is reflected off of a dichroic beamsplitter [30] and focused through lens $\mathrm{L}_{1}$ into the capillary. The windowed-flange $(\mathrm{W})$ allows the transmission of light into and out of the capillary while maintaining the capability of pressurizing or vacuuming the waveguide. The other end of the waveguide is fitted with a T-fitting to allow the introduction or evacuation of test gases at that end of the guide as well as measurement of residual pump-power through a window in the third leg of the T-fitting. Backscattered Raman light passes with little attenuation through the dichroic beamsplitter (BS) and then through an aperture (A) and a long-pass Raman edge-filter (F) before being focused by $\mathrm{L}_{2}$ into a grating spectrometer (JY iHR550). $\mathrm{L}_{1}$ is separated from the capillary by its focal length $\left(f_{1}\right)$ and $\mathrm{L}_{2}$ is spaced a focal distance $\left(f_{2}\right)$ from the spectrometer. The separation between the two lenses is $f_{1}+f_{2}$. The linear angle collected from the capillary $\left(\theta_{\mathrm{c}}\right)$ is magnified by the two-lens system, and the signal entering the spectrometer will have an angular spread $\theta_{\mathrm{s}}$ where

$$
\theta_{s}=\frac{f_{1}}{f_{2}} \theta_{c} .
$$

Figure 4 shows that all modes with $\theta_{\mathrm{c}}<90^{\circ}$ propagate, and consequently some output will be lost in any detection system with limited angular acceptance. The pump beam is generally introduced into the capillary with a shallow launch angle to propagate as a low-order waveguide mode, and as such, will have very little propagation loss (see Fig. 4). For backscattered Stokes radiation, the differential Stokes power generated at each position $d z$ along the waveguide is

$$
d P_{s}=\sigma \rho P_{p} e^{-\alpha(\theta) z} d \Omega d z=2 \pi \sigma \rho P_{p} e^{-\alpha(\theta) z} d z \sin \theta d \theta .
$$

Therein, $d P_{s}$ is the Stokes power generated in a length $d z$ and in solid angle $d \Omega, \sigma$ is the Raman cross section of the scattering medium, $\rho$ is the molecular density, $P_{p}$ is the pump laser power, and $d \theta$ is an increment of linear collection angle. Integrating Eq. (15) over length and angle allows us to determine the total Stokes Raman power that will be collected from the Raman-output end of the waveguide:

$$
P_{s}=\int_{0}^{\theta_{c}} \int_{0}^{l} 2 \pi \sigma \rho P_{p} e^{-\alpha(\theta) z} d z \sin \theta d \theta .
$$

These theoretical predictions were verified using the system shown in Fig. 6. The capillary was a $2 \mathrm{~m}$ long, silver-lined, $530 \mu \mathrm{m}$ I.D. waveguide filled with pressurized methane (200 psig) gas. The fiber was held straight to avoid losses associated with bending [1,4,6,19,23,24]. The pump laser was a Coherent I-70 $514.5 \mathrm{~nm}$ argon-ion laser producing a $\sim 100 \mathrm{~mW} \mathrm{TEM}_{00}$ beam. $\mathrm{L}_{1}$ and $\mathrm{L}_{2}$ had focal lengths of $20 \mathrm{~mm}$ and $75 \mathrm{~mm}$, respectively, providing a magnification of $3.75 \mathrm{X}$, accompanied by a 0.267 angular demagnification. The spectrometer collects light up to f/6.4 (4.5 half-angle), and the 3.75X magnification allows collection of waveguide linear angles up to about $17^{\circ} \mathrm{lim}$ - 
ited by the spectrometer's angular aperture. Raman output power was measured along with capillary output angle by using the variable aperture (Fig. 6) to measure the output angle and a cooled single-channel silicon photodiode (EO Systems S-025/TE2) to measure Raman power. The Stokes methane line at $2917 \mathrm{~cm}^{-1}(605.4 \mathrm{~nm})$ was measured while varying the aperture's diameter to determine the angular spread of Raman output. Figure 7 plots this collected Raman power along with the predicted results [Eq. (16)]. The simulated Stokes power has been normalized to account for spectrometer efficiency, detector efficiency, and pump power. Figure 7 shows excellent agreement between the experimental and simulation results up to the maximum angular acceptance of the detection system. Figure 7 shows significant contributions to the Raman output for $\theta_{c}$ as great as $15^{\circ}$, where $\alpha$ [calculated from Eqs. (4), (10), (11), and (13)] is as great as $30 \mathrm{~m}^{-1}$. We note that Raman photons produced near the waveguide-end (closest to W, Fig. 6) will couple into these extremely lossy high-order modes, but will experience small total transmission losses when propagating a short distance through the guide before emerging into free space. Figure 7 highlights the role of distributed Raman scattering. Light rays that propagate at large values of $\theta_{c}$ have large absorption coefficients and produce significant Raman power output only from regions near the guide's output. Nonetheless, the contributions from these rays are noticeable even up to $\theta_{c}=15^{\circ}$, indicating the relevancy of accurate higher-order mode-loss calculations in predicting Raman power. Traditional loss estimates in waveguides of this type are generally simplified to predict a value of loss coefficient $\alpha$ proportional to $u_{1 m}{ }^{2} / a^{3}$ $[17,18]$. When the exact losses of higher-order modes are considered, this estimate becomes unsuitable and can result in significant error in the total predicted Raman output power. We have predicted Raman output powers based on the traditional simplified approach (not shown) alongside our new approach, and find as much as 50\% error for total Raman power calculated using the loss coefficients derived by Marcatili and Schmeltzer [17].

Various possible waveguide configurations were simulated using the algorithm described. The effects of changing waveguide material, diameter, and length on the total collected Raman power were predicted. These predictions

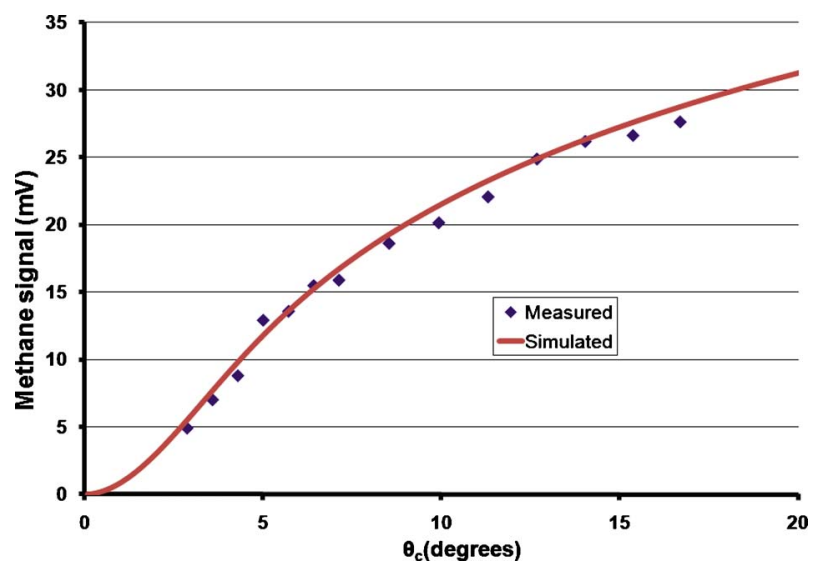

Fig. 7. (Color online) Simulated and measured Stokes Raman power collected in a metallized waveguide. allow the optimization of capillary parameters for a particular light collection system.

Figure 8(a) shows that Stokes radiation exists for all $\theta_{c}<90^{\circ}$, and radiation traveling at large angles with respect to the capillary's axis experiences large losses (see Fig. 4), but still radiation contributes to the Stokes output. The results shown in Fig. 8(a) have important consequences in the design of a detection system for maximum collection of Stokes radiation. A spectrometer or other detection system is characterized in terms of its étendue $\left(A \Omega_{s}\right)$, where $A$ is the area of the entrance slits or active detection surface, and $\Omega_{s}$ is the detector's collection solid angle. Imaging and magnification of the Stokes output from the capillary can change the size of the Stokes beam or change its solid angle, but the étendue of the Stokes beam is unchanged. Thus the detection system's étendue determines how much of the capillary Stokes output is captured. For a circular detector (radius $b$ ) with a small collection angle $\left(\theta_{s}\right)$, étendue $\approx\left(\pi b \theta_{s}\right)^{2}$. Because imaging does not change étendue, $b \theta_{s}=a \theta_{c}$, where $a$ is the radius of the capillary and $\theta_{c}$ is the angle of the Stokes radiation that is collected from the capillary.

Figure 8(b) shows how the collected Stokes power is affected by the detection system collection parameter. Figure 8(b) assumes that the collection optics are designed so that the image of the entire capillary is accepted by the detection system. For a large $b \theta_{s}$, the large capillary produces a larger detected Stokes signal than the smaller capillary, but for a detection system with small $b \theta_{s}$ (see inset Fig. 8(b)) this is not true. At very small values of $b \theta_{s}$, the smallest diameter capillary produces the largest signal. The reason for this somewhat unusual result is related to the fact that at a given propagation angle $\left(\theta_{c}\right)$, losses are smaller in the larger bore size capillary (see Fig. 4). Therefore, a smaller collection angle is required to collect all low-loss rays propagating in a smaller capillary than for a larger capillary. Figure 8 then allows us to choose a capillary cell diameter for a given detection system with the most throughput.

For example, consider two capillaries of length $0.1 \mathrm{~m}$ and diameters $2 a=320 \mu \mathrm{m}$ and $800 \mu \mathrm{m}$. If "low loss" is defined as $\alpha l<1.0$, Fig. 4 shows that in the smaller capillary, a collection angle of $6.4^{\circ}\left[2 b \theta_{s}=2 a \theta_{c}=(0.320)(6.4)\right.$ $=2.0$ degrees-mm $]$ collects all the low-loss rays $(\alpha$ $<10 \mathrm{~m}^{-1}$ ). In the larger capillary, the corresponding collection angle for $\alpha<10 \mathrm{~m}^{-1}$ is $10.3^{\circ}\left[2 b \theta_{s}=(0.800)(10.3)\right.$ $=8.2]$. Thus, for $2.0<2 b \theta_{s}<8.2$, the smaller capillary collects all low-loss rays but the larger capillary does not. Figure 8(b) shows that in this range, the smaller capillary collects more Stokes radiation. For larger values of $b \theta_{s}$, the Stokes output from the large capillary dominates.

Figure 9 shows the effect of waveguide length on Stokes power collection. For detection equipment with limited collection angles, a significant increase in Stokes power occurs with increased capillary length. We also observe that increasing waveguide lengths offers continuously increased output with diminishing marginal returns because of the backscattered nature of the collection. More simply, an infinitely long waveguide is only marginally better than a $\sim 10 \mathrm{~m}$ long guide.

Finally, we look at the difference between two metals as waveguide linings. Figure 10 compares two otherwise 


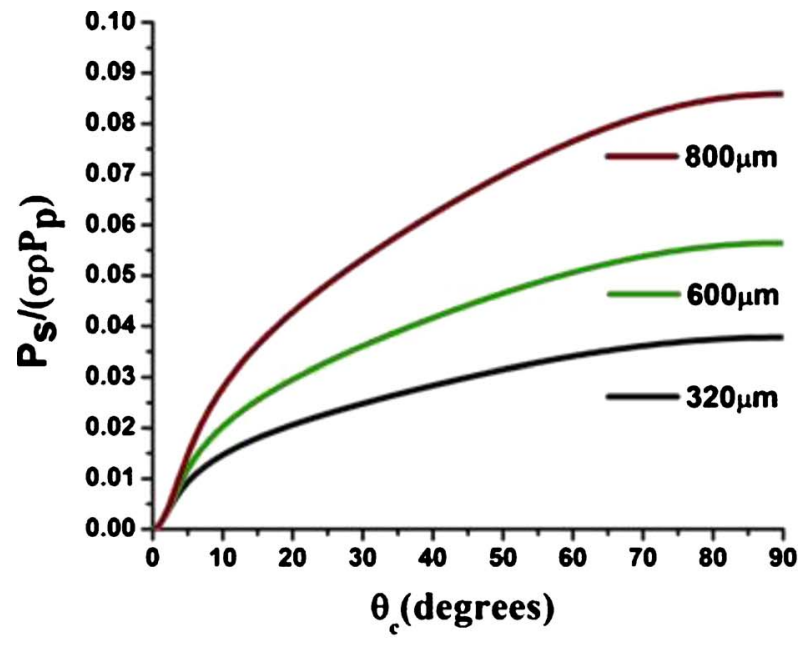

(a)

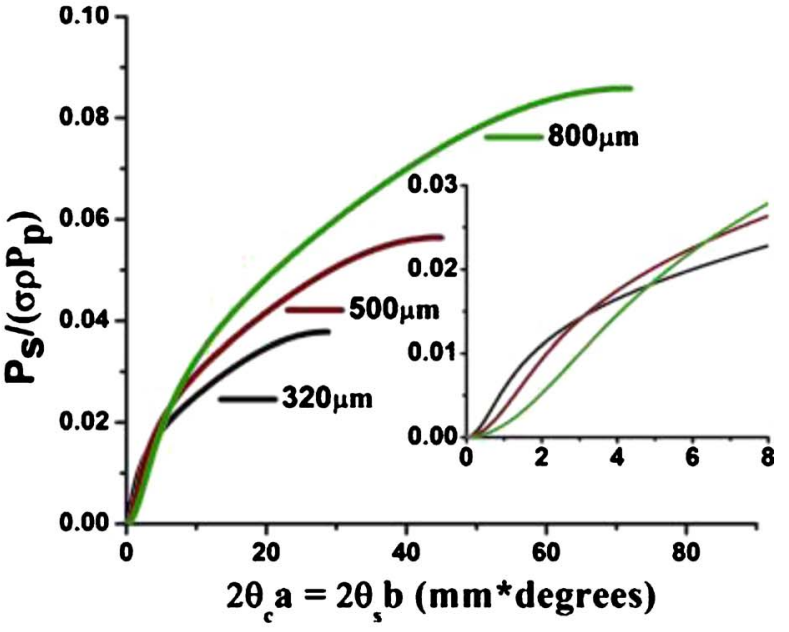

(b)

Fig. 8. (Color online) (a) Predicted, normalized Stokes power output as a function of waveguide diameter; silver coated capillary, $1 \mathrm{~m}$ long, $\lambda=584.5 \mathrm{~nm}$. (b) Predicted, normalized power as a function of $2 \theta_{c} a$ (see text).

identical guides, one lined with aluminum and one with silver. In rough terms, the Raman output will be deter-

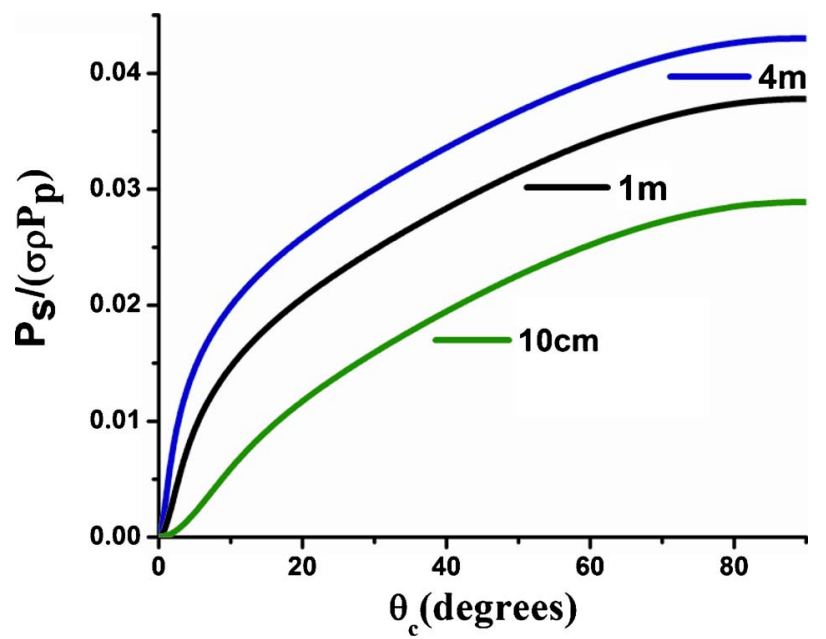

Fig. 9. (Color online) Effects on Stokes power with varying waveguide length (backscattering); silver-lined capillary, $2 \mathrm{a}$ $=320 \mu \mathrm{m}, \lambda_{\text {pump }}=514.5 \mathrm{~nm}$, nitrogen Raman, $\lambda_{\text {Stokes }}=584.5 \mathrm{~nm}$.

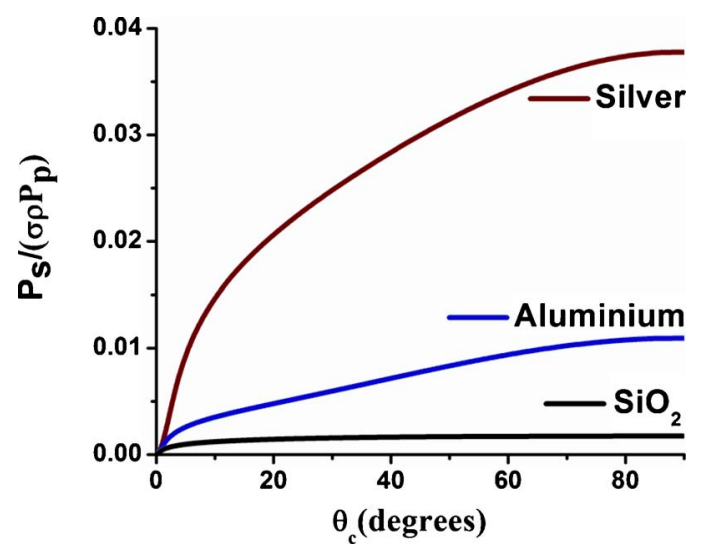

Fig. 10. (Color online) Waveguide material comparison ; $l$ $=1 \mathrm{~m}, 2 a=320 \mu \mathrm{m}, \lambda_{\text {pump }}=514.5 \mathrm{~nm}$, nitrogen Raman, $\lambda_{\text {Stokes }}$ $=584.5 \mathrm{~nm}$. mined by the inverse of the real part of the metal's complex index of refraction at the wavelength of interest. Therefore, silver $(\nu=0.121+3.61 \mathrm{i}$ at $584.5 \mathrm{~nm})$ is a better collector in the visible than aluminum $(\nu=1.12+7.085 \mathrm{i}$ at $584.5 \mathrm{~nm}$ ) [18]. Other authors have shown that an appropriately chosen additional inner dielectric lining will increase the guide-wall reflectivity, and thus will increase the Raman output from a gas-filled capillary. Matsuura et al. [6] and Nubling and Harrington [26] have shown that a properly sized dielectric lining applied to the metal-lined capillary will reduce the modal losses uniformly. This will lead to changes in the amplitudes of the Raman powers shown in Fig. 10 but only small changes in the shape of these curves. The detailed consideration of the influence of dielectric/metal-lined capillaries on total Raman output is beyond the scope of this paper.

For comparison, Fig. 10 also shows Raman output for an unlined (no metal) dielectric (refractive index 1.5) [14]. At any propagation angle, the dielectric guide has higher losses than either of the metal guides, and consequently is capable of producing much less Raman output.

\section{CONCLUSIONS}

Metal-lined and metal/dielectric-lined capillary waveguides are useful devices for both power delivery and light collection applications. Although the basic equations underlying the operating characteristics of these waveguides have been known for decades, there has been a lack of quantitative analysis of higher-order modes predicted by these equations. Understanding the multimode nature of these waveguides is necessary for optimizing their use in collecting scattered light. We have provided a mathematical approach for accurately calculating or estimating metallic-waveguide losses for large numbers of propagating optical modes. Although the highest-order modes exhibit extremely high loss, these modes contribute to collection efficiency because propagation distances can be short for analytes near the output end of the guide. (Conversely, the low-loss, low-order modes can be used for efficient power delivery.) 
By extending the analytical approach taken by earlier researchers and eliminating assumptions correct only for low-order mode calculations, we were able to accurately estimate mode-losses for the higher-order modes propagating at large angles in the metal waveguide. These estimates were experimentally validated with excellent agreement to theory. Although our work dealt only with the linearly polarized $E H_{1 m}$ modes, evaluation of the other types of modes would be possible given the same methods shown here. While some additional computational complexity would be required, along with consideration for the excitation amplitudes of various mode types for a given source with a particular polarization ratio, it would be possible to expand this work to all mode types following roughly the same algorithm. The methods utilized herein may also be applicable to the study of dielectric-lined metal waveguides with the addition of boundary conditions at each layer interface and significantly more mathematical complexity. Practically speaking, such analysis could yield further improvements in collectible Raman power, given solutions for optimized dielectric over-coat thickness. The addition of an inner dielectric layer should also reduce bending losses in the fiber, which could make it possible to coil the sensor system into a smaller package. Once such optimal coatings have been designed, fabrication difficulties in maintaining coating tolerances for extremely thin dielectric layers on the inside of capillaries can be addressed. Given an appropriate understanding of the mode properties of metallic capillary waveguides, researchers will continue to discover new applications for their implementation in science and technology.

\section{ACKNOWLEDGMENTS}

The authors thank Mr. Stephen Biedrzycki for many useful discussions.

This technical effort was performed in support of the National Energy Technology Laboratory's research in Energy Systems and Dynamics under RDS contract DEAC26-04NT41817 and RES contract DE-FE0004000.

\section{REFERENCES}

1. Y. Abe, Y. Matsuura, Y. Shi, Y. Wang, H. Uyama, and M. Miyagi, "Polymer-coated hollow fiber for $\mathrm{CO} 2$ laser delivery," Opt. Lett. 23, 89-90 (1998).

2. Y. Abe, Y. Shi, Y. Matsuura, and M. Mitsunoba, "Flexible small-bore hollow fibers with an inner polymer coating," Opt. Lett. 25, 150-152 (2000).

3. Y. Komachi and H. Sato, "Raman probe using a single hollow waveguide," Opt. Lett. 30, 2942-2944 (2005).

4. Y. Matsuura, K. Hanamoto, S. Sato, and M. Miyagi, "Hollow-fiber delivery of high-power pulsed Nd:YAG laser light," Opt. Lett. 23, 1858-1860 (1998).

5. Y. Matsuura, M. Saito, M. Miyagi, and A. Hongo, "Loss characteristics of circular hollow waveguides for incoherent infrared light," J. Opt. Soc. Am. A 6, 423-427 (1989).

6. Y. Matsuura, G. Takada, T. Yamamoto, Y. Shi, and M. Mitsunobu, "Hollow fibers for delivery of harmonic pulses of Q-switched Nd:YAG lasers," Appl. Opt. 41, 442-445 (2002).

7. Y. Matsuura, Y. Shi, Y. Abe, M. Yaegashi, G. Takada, S. Mohri, and M. Miyagi, "Infrared-laser delivery system based on polymer-coated hollow fibers," J. Opt. Laser Tech. 33, 279-283 (2001).

8. Y. Matsuura and M. Miyagi, "Hollow optical fibers for ultraviolet and vacuum ultraviolet light," IEEE J. Sel. Top. Quantum Electron. 10, 1430-1434 (2004).

9. M. P. Buric, K. P. Chen, J. Falk, and S. D. Woodruff, "Metallined capillaries for efficient Raman gas sensing," Conference on Lasers and Electro-Optics (Optical Society of America, 2010), paper CFA5.

10. M. Buric, K. P. Chen, J. Falk, and S. D. Woodruff, "Improved sensitivity gas detection by spontaneous Raman scattering," Appl. Opt. 48, 4424-4429 (2009).

11. M. Buric, K. Chen, J. Falk, and S. Woodruff, "Enhanced spontaneous Raman scattering and gas composition analysis using a photonic crystal fiber," Appl. Opt. 47, 4255-4261 (2008).

12. M. Buric, K. P. Chen, J. Falk, and S. D. Woodruff, "Improved sensitivity spontaneous Raman scattering multi-gas sensor," Conference on Lasers and Electro Optics (Optical Society of America, 2009). Available from IEEE Xplore Lasers and Electro-Optics, 2009. CLEO 2009.

13. Doko Engineering, capillary waveguide specifications. Downloaded from http://do-ko.jp/specs.html (June 9, 2010).

14. S. D. Schwab and R. L. McCreery, "Remote, longpathlength cell for high-sensitivity Raman spectroscopy," Appl. Spectrosc. 41, 126-130 (1987).

15. M. Buric, K. Chen, J. Falk, R. Velez, and S. Woodruff, "Raman sensing of fuel gases using a reflective coating capillary optical fiber," SPIE Symposium on Defense Security and Sensing (SPIE, 2009).

16. J. A. Stratton, Electromagnetic Theory (McGraw-Hill, 1942). p. 524.

17. E. Marcatili and R. Schmeltzer, "Hollow metallic and dielectric waveguides for long distance optical transmission and lasers," Bell Syst. Tech. J. 43, 1783-1809 (1964).

18. J. P. Crenn, "Optical study of the EH11 mode in a hollow circular oversized waveguide and Gaussian approximation of the far-field pattern," Appl. Opt. 23, 3428-3433 (1984).

19. J. A. Harrington, "A review of IR transmitting, hollow waveguides," Fiber Integr. Opt. 19, 211-217 (2000).

20. M. Miyagi and S. Kawakami, "Design theory of dielectriccoated circular metallic waveguides for infrared transmission," J. Lightwave Technol. LT-2, 116-126 (1984).

21. P. Bhardwaj, O. J. Gregory, C. Morrow, and K. Burbank, "Performance of dielectric-coated monolithic hollow metallic waveguide," Mater. Lett. 16, 150-156 (1993).

22. Y. Matsuura and M. Miyagi, "Er:YAG, CO, and CO2 laser delivery by ZnS-coated Ag hollow waveguides," Appl. Opt. 32, 6598-6601 (1993)

23. Y. Shi, Y. Wang, Y. Abe, Y. Matsuura, M. Miyagi, S. Sato, M. Taniwaki, and H. Uyama, "Cyclic olefin polymer coated silver hollow glass waveguides for the infrared," Appl. Opt. 37, 7758-7762 (1998).

24. J. A. Harrington, Infrared Fibers and Their Applications (SPIE Press, 2004).

25. E. Snitzer, "Dielectric waveguide modes," J. Opt. Soc. Am. 51, 491-498 (1961).

26. R. K. Nubling and J. A. Harrington, "Launch conditions and mode coupling in hollow-glass waveguides," Opt. Eng. 37, 2454-2458 (1998).

27. M. Abramowitz and I. A. Stegun, eds., Handbook of Mathematical Functions with Formulas, Graphs, and Mathematical Tables (Dover, 1972), pp. 370-374.

28. Y. W. Shi, K. Ito, Y. Matsuura, and M. Miyagi, "Multiwavelength laser light transmission of hollow optical fiber from the visible to the mid-infrared," Opt. Lett. 30, 2867-2869 (2005).

29. G. Turrell and J. Corset, Raman Microscopy, Developments and Applications (Elsevier, 1996).

30. Semrock beamsplitter data sheet. Downloaded from http:// www.semrock.com/Catalog/RamanEdgeDichroic.htm (July $23,2008)$. 